\title{
VALOR DO EEG NA CARACTERIZAÇÃO E PROGNÓSTICO DE PATOLOGIAS NEUROLÓGICAS EM RECÉM-NASCIDOS PREMATUROS
}

\author{
MAGDA LAHORGUE NUNES*, JADERSON COSTA DA COSTA**, \\ LETÍCIA TAUFER ***, CLAUDIA MARISA DURO DA SILVEIRA***
}

\begin{abstract}
RESUMO - A importância do EEG na investigação de patologias neurológicas no periodo neonatal tem sido discutido com frequência na atualidade, pois muitas vezes este é o único meio de avaliar a função cerebral em recém-nascidos (RN) com patologias graves ou sob efeito de medicações. O presente estudo foi realizado com $85 \mathrm{RN}$ prematuros que apresentaram patologias neurológicas no período neonatal e foram submetidos a avaliaçāo neurológica e cletrencefalográfica (EEG ou polissonografia -PS). Foram relacionadas alterações do EEG, patologias e prognóstico. Os EEGs foram classificados scgundo as alteraçõcs da atividade de base, presença de atividade paroxística, organização dos estágios do sono e "maturidade". A patologia mais frequente foi asfixia perinatal $(40 \%)$, seguida de hemorragia intra-ventricular (HIV,16\%). A queixa mais frequente na indicaçāo do exame foi apnéia ( $71 \%$ ), seguida de convulsões (19\%). Foram considerados normais $55 \%$ dos exames solicitados com queixa exclusiva de apnéia; dos exames solicitados por convulsōes somente $31 \%$ foram normais. A alteração do EEG mais frequentemente encontrada nos RN com asfixia, HIV e desnutriçāo intra-uterina foi a "imaturidade" da atividade elétrica cerebral. Nos RN com convulsões as alterações mais frequentes do EEG foram atividade paroxística anormal e "imaturidade". Os RN com infeç̧ão do sistema nervoso central apresentaram diversas alteraçōes no EEG. Os achados de EEG mais correlacionados a mau prognóstico foram isoeletricidade e atividade paroxística anormal (100\% dos casos com presença de ondas agudas positivas).
\end{abstract}

PALAVRAS-CHAVE: recém-nascido, prematuridade, EEG, polissonografia.

\section{Relationship of EEG, neurological diseases and follow-up in preterm newborns}

SUMMARY - The importance of the EEG for the investigation of neurological diseases in the neonatal period has been largely discussed, since it is often the only way to approach cerebral function in newborns with severe pathologies or under drug effect. The present study was carricd out with 85 newborns (NB) who presented perinatal dysfuntions and were submitted to neurological and electroencephalogram (EEG) or polysomnography (PS) evaluation. EEG/PS alterations, pathologies and prognosis were reported. The EEG were classified according to basal activity alterations, presence of paroxysmal activity and sleep stages organization and maturity. The most frequent pathology was perinatal asphyxia (40\%) followed by intraventricular hemorrhage (HIV, 16\%). The most frequent complaint for exam indication was apnea (71\%) followed by convulsion (19\%). Fifty-five percent of the exams exclusively required because apnea complaint were considered normal and out of all exams required because seizures only $31 \%$ were normal. The EEG alteration most frequently related to perinatal asphyxia, HIV and intrauterine growth delay was immaturity and in the NB with seizures immature EEG and abnormal paroxysms. Many different alterations were registered in the NB with nervous system infection. The EEG findings more correlated with unfavorable prognosis were isoelectricity and abnormal paroxysmal activity including positive sharp waves (100\%).

KEY WORDS: newborn, premature, EEG, polysomnography.

Serviço de Neurologia e Laboratório de Neurofisiologia Clínica do Hospital São Lucas e Faculdade de Medicina da Pontífica Universidade Católica do Rio Grande do Sul (PUCRS), Porto Alegre: *Professora Auxiliar de Ensino de Neurologia da Faculdade de Medicina da PUCRS, Chefe do Laboratório de Neurofisiologia Clínica do Hospital São Lucas da PUCRS; **Professor Titular de Neurologia da Faculdade de Medicina da PUCRS, Chefe do Serviço de Neurologia do Hospital São Lucas da PUCRS; ***Acadêmica da Faculdade de Medicina da PUCRS. Aceite: 13-abril-1995. 
Os cuidados intensivos neonatais têm apresentado expressivo desenvolvimento nos últimos 30 anos, cada vez mais possibilitando a sobrevida de recém-nascidos (RN) com idade gestacional (IG) às vezes até inferior a 26 semanas. A maior expectativa de sobrevida para o RN com peso inferior a 1500 gramas resultou na ampliaçăo da populaçāo de RN, surgindo a necessidade de aprimoramento dos métodos jả existentes para avaliação da função cerebral nestes bebês. A importancia do EEG na investigação de patologias neurológicas no período neonatal é assunto que tem sido revisado com frequência ${ }^{1,5,6,8,9-11}$, já que este é um método não invasivo e de baixo custo que atua como instrumento de suporte e complementar da avaliação clínica, sendo sensível na detecção precoce e previsāo do prognóstico de disfunçōes cerebrais no RN.

O objetivo deste estudo é relacionar em RN prematuros as alterações observadas no EEG/PS realizado no período neonatal com a existência de doença neurológica e prognóstico.

\section{MATERIAL E MÉTODOS}

O presente estudo foi realizado em 85 RN prematuros com idade gestacional inferior a 37 semanas, internados na UTI Neonatal do Hospital São Lucas da PUCRS em período de 10 anos (1982-1993), que apresentaram patologias neurológicas no período neonatal e foram submetidos a avaliação neurológica e EEG ou PS.

Os exames foram realizados em aparelho de 13 ou 16 canais com eletrodos colocados pelo Sistema 1020 modificado para $\mathrm{RN}^{2,7}$. As PS constavam de 8 ou 11 canais de EEG, 2 canais de EOG, EMG, monitorização de fluxo aéreo nasal e movimentos respiratórios abdominais e ECG. Os exames foram realizados em sono espontâneo nas fases REM e NREM com duração de 20-40 minutos (EEG) e 50-60 minutos (PS) e foram classificados considerando a proposta de Lombroso $0^{4} \mathrm{em}$ três grupos. Grupo I, alteraçōes da atividade de base: os EEGs apresentavam padrões de isoeletricidade (atividade inferior a 5 microvolts contínua durante o registro e nāo reatividade), baixa voltagem (atividade entre 5-15 microvolts durante todo o traçado), assimetria (atenuação persistente de voltagem em determinada região do escalpo) e surto-supressão (períodos de inatividade com 2-10 segundos interrompidos por surtos de alta voltagem com duração de 1-10 segundos). Grupo II, presença de atividade paroxística: neste grupo foi considerada somente atividade paroxística anormal interictal ou ictal (pontas, atividade pseudo-ritmica, ondas agudas positivas). Grupo III, distúrbios na organizaçāo dos estágios do sono e maturidade: neste grupo foram incluidos os registros que mostravam padrões bioelétricos no EEG/PS compativeis com idade concepcional (IC) inferior à obtida pelo exame físico do RN (diferença superior a duas semanas). A este achado denominamos de EEG "imaturo", "dismaturo" ou "imaturidade bioelétrica". Foi considerado neste estudo somente o primeiro EEG/PS realizado. Para definição de "imaturidade" persistente ou transitória da atividade elétrica cerebral foram considerados, quando cxistentes, EEGs subsequentes realizados em intervalo superior a uma semana. Assim quando a "imaturidade bioelétrica" inicialmente diagnosticada foi revertida nos EEG/PS subsequentes realizados até a 44-46a. semana de IC ela foi denominada como "transitória"; caso ela se mantivesse além desta faixa nós a consideramos "persistente"2,4,5,7. Foram relacionadas as alterações do EEG com as patologias diagnosticadas nos RN.

Nos casos em que foi possivel obter seguimento, relacionamos as alterações do EEG com o prognóstico dos RN. O seguimento destes RN foi efetuado através de consultas ambulatoriais e/ou visitas domiciliares e seu desenvolvimento neuropsicomotor foi avaliado utilizando o teste de Denver ${ }^{3}$. Consideramos seguimento anormal naqueles RN que desenvolveram um ou mais dos seguintes itens: sinais focais e/ou deficitários ao exame neurológico, epilepsia, atraso no desenvolvimento neuropsicomotor ou que evoluíram para óbito durante o primeiro ano de vida.

\section{RESULTADOS}

A IG dos RN variou de 28-34 semanas, 37 eram do sexo feminino e 48 do masculino. Sessenta RN (71\%) foram submetidos a EEG/PS por apresentarem apnéias, dos quais $38(63 \%)$ apresentavam apnéias associadas a outros sintomas ou decorrentes de outras patologias e 21 (37\%) apresentavam exclusivamente apnéias da prematuridade (como queixa isolada); 16 (19\%) apresentaram crises convulsivas e $2(3 \%)$ tremores. A patologia de base detectada com maior incidência foi a asfixia perinatal (39\%), seguida por hemorragia intraventricular (HIV,16\%), desnutrição intra-útero (12\%) 
Tabela I. Correlação entre EEG/PS neonatal, manifesfação clínica paraxística e seguimento do RN premature.

\begin{tabular}{ccccc}
\hline Manifest. clin. parox. & Apnéia + & Apnéia & Convulsão & Tremor \\
EEG & $\mathrm{n}=38$ & $\mathrm{n}=32$ & $\mathrm{n}=16$ & $\mathrm{n}=2$ \\
\hline Normal & $15(2 \mathrm{~N} / 2 \mathrm{~A} / 1 \mathrm{P})$ & $12(3 \mathrm{~N} / 3 \mathrm{~A} / 6 \mathrm{P})$ & $5(2 \mathrm{~N} / \mathrm{A} / 2 \mathrm{P})$ & $1(\mathrm{P})$ \\
Paroxístico & $8(\mathrm{~A} / 7 \mathrm{P})$ & $5(\mathrm{~N} / \mathrm{A} / 3 \mathrm{P})$ & $7(4 \mathrm{~A} / 3 \mathrm{P})$ & 0 \\
BV & $2(\mathrm{P} / \mathrm{P})$ & 0 & $1(\mathrm{P})$ & $1(\mathrm{~N})$ \\
Isoelétrico & 0 & 0 & 0 & 0 \\
Assimétrico & 0 & $1(\mathrm{P})$ & 0 & 0 \\
OAP & $2(\mathrm{~A} / \mathrm{P})$ & 0 & $2(\mathrm{~A} / \mathrm{P})$ & 0 \\
Imaturidade & $11(2 \mathrm{~N} / 2 \mathrm{~A} / 7 \mathrm{P})$ & $3(\mathrm{~N} / 2 \mathrm{P})$ & $3(\mathrm{~A} / 2 \mathrm{P})$ & 0 \\
IT & $3(\mathrm{~N} / 2 \mathrm{P})$ & $1(\mathrm{P})$ & 0 & 0 \\
IP & $1(\mathrm{P})$ & 0 & $1(\mathrm{P})$ & 0 \\
\hline
\end{tabular}

BV, baixa voltagem; OAP, onda aguda positiva; IT, imaturidade transitória; IP, imaturidade persistente; Apnéia + , apnéia associada a outras queixas ou patologias; $N$, seguimento normal; $A$, seguimento anormal; $P$, seguimento perdido.

e infecção do sistema nervoso central (SNC, 9\%). A idade concepcional (IC) na ocasião da realização do primeiro EEG/PS variou de 30-44 semanas.

Relacionando as alteraçōes do EEG/PS com a indicaçāo do exame observamos que: 55\% dos exames solicitados com a queixa exclusiva de apnéia foram normais; nos exames solicitados por apnéia associada a outros distúrbios 39\% foram normais; nos solicitados para investigaçāo de convulsōes somente $31 \%$ foram normais (Tabela 1). Relacionando patologias de base com EEG observamos que $66 \%$ dos RN com asfixia apresentaram EEG anormal, a alteração mais freqüentemente encontrada sendo "imaturidade" (42\%). Nos RN com HIV 64\% dos exames foram anormais e as alterações mais freqüentemente encontradas foram imaturidade (67\%) e presença de ondas agudas

Tabela 2. Correlaçāo entre EEG/PS neonatal, patologia neurológica e seguimento de RN prematurux.

\begin{tabular}{|c|c|c|c|c|c|c|c|c|c|c|c|c|}
\hline EEG & $n=34$ & $n=14$ & $\mathrm{n}=14$ & $\begin{array}{l}\text { INF } \\
\text { SNC } \\
n=8\end{array}$ & $\mathrm{n}=2$ & $\begin{array}{c}\text { Lues } \\
\text { Cong. } \\
\mathrm{n}=2\end{array}$ & $\mathrm{n}=2$ & $\begin{array}{l}\text { Fipogli- } \\
\text { cemia } \\
n=2\end{array}$ & HIV + & $\begin{array}{l}\text { S. Abst. } \\
n=1\end{array}$ & $\begin{array}{c}\text { Tocotrau- } \\
\text { mitismo } \\
n=1\end{array}$ & $\begin{array}{c}\text { Embolia } \\
\text { Intrauterina } \\
n=1\end{array}$ \\
\hline Normul & $\begin{array}{c}15 \\
(3 \mathrm{~N} / 2 \mathrm{~A} / 10 \mathrm{P})\end{array}$ & $\begin{array}{c}5 \\
(2 N / 3 P)\end{array}$ & $1(P)$ & $\begin{array}{c}5 \\
(\mathrm{~A} / 4 \mathrm{P})\end{array}$ & $l(N)$ & 0 & $2(P)$ & $1(P)$ & 0 & 0 & $I(P)$ & 0 \\
\hline Paroxístico & $\begin{array}{c}9 \\
(\mathrm{~N} / 2 \mathrm{~A} / 6 \mathrm{P})\end{array}$ & $2(\mathrm{P} / \mathrm{P})$ & $1(\mathrm{~N})$ & $1(\mathrm{~N})$ & 0 & 0 & $I(A)$ & 0 & 0 & 0 & 0 & 0 \\
\hline BV & $2(2 P)$ & $1(P)$ & $1(\mathrm{~N})$ & $1(\mathrm{~A})$ & 0 & $2(2 P)$ & 0 & 0 & 0 & $1(\mathrm{~N})$ & 0 & 0 \\
\hline Isoelêtrico & 0 & 0 & 0 & 0 & 0 & 0 & 0 & 0 & 0 & 0 & 0 & $1(A)$ \\
\hline Assimétrico & 0 & 0 & 0 & 0 & 0 & 0 & 0 & 0 & 0 & 0 & 0 & 0 \\
\hline OAP & $2(A)$ & 3 & $1(\mathrm{~A})$ & 0 & 0 & 0 & 0 & 0 & 0 & 0 & 0 & 0 \\
\hline $\begin{array}{l}\text { Imaturidade } \\
\text { bioelétrica }\end{array}$ & $\begin{array}{c}7 \\
\text { (2N/A/4P) }\end{array}$ & $\begin{array}{c}3 \\
(\mathrm{~A} / 2 \mathrm{P})\end{array}$ & $3(P)$ & $1(\mathrm{~A})$ & 0 & 0 & 0 & 0 & $1(\mathrm{P})$ & 0 & 0 & 0 \\
\hline IT & $I(N)$ & $1(\mathrm{P})$ & $I(P)$ & 0 & 0 & 0 & 0 & 0 & 0 & 0 & 0 & 0 \\
\hline IP & 0 & $2(2 P)$ & I (P) & 0 & 0 & 0 & 0 & 0 & 0 & 0 & 0 & 0 \\
\hline
\end{tabular}

BV, baixa voltagem; OAP, onda aguda positiva; IT, imaturidade transitória; IP, imaturidade persistente; HIV, hemorragia intraventricular, PIG, pequeno para idade gestacional; INF SNC, infecção do sistema nervoso central; HSA, hemorragia subaracróidea; HIV +, soropositividade para vírus da imunodeficiência humana; S. ABST, síndrome de abstinência; $N$, seguimento normal; A, seguimento anormal; P, seguimento perdido. Alguns EEGs apresentavam mais de uma alteração. 
positivas (21\%). Todos os pacientes com HIV grau III apresentaram EEG/PS anormais. Somente um RN que apresentou desnutrição intra-útero (pequeno para a idade gestacional, PIG), teve EEG normal e a alteração mais freqüentemente encontrada neste grupo foi a "imaturidade". Nos RN com infecção do SNC 63\% dos exames foram normais e nos restantes foram diversas as alteraçōes detectadas (Tabela 2).

Foi possível obter seguimento em $29 \mathrm{RN}(32,5 \%)$, que variou de 3 meses a 11 anos (média de 4,5 anos). Treze RN foram considerados normais em seguimento que variou de 3 meses a 11 anos (média de 5,4 anos); destes, 7 (53\%) apresentaram EEG inicial normal. Dezesseis RN foram considerados anormais com seguimento que variou de 1 ano e 2 meses a 8 anos (média de 4 anos); destes 5 evoluíram para óbito no primeiro ano de vida. Neste grupo, 5 RN (31\%) apresentaram EEG normal.

Dos 40 prematuros que apresentaram EEG normal foi obtido seguimento em 12 (30\%): 7 foram considerados normais, 2 apresentaram discreto atraso no desenvolvimento neuropsicomotor, um dificuldade escolar associada a distúrbio da atenção e 2 evoluíram para óbito no período neonatal, sendo a causa provavelmente relacionada ao peso muito baixo de nascimento (600 e 900 gramas). Os dois RN que evoluiram para óbito não desenvolveram complicações neurológicas (em ambos, o exame foi indicado por queixa exclusiva de apnéia).

O seguimento mostrou que somente dois RN desenvolveram epilepsia e em ambos também foi constatado atraso no desenvolvimento neuropsicomotor. No período neonatal, um apresentou crises convulsivas provavelmente relacionadas a asfixia perinatal e o segundo somente apnéias, ambos com EEG alterado (atividade paroxística anormal).

Os achados eletrencefalográficos que mais se correlacionaram com mau prognóstico foram isoeletricidade e ondas agudas positivas (100\%).

Comparando resultado do EEG inicial com prognóstico, apesar de termos observado uma tendência de EEG inicial normal correlacionar-se com bom prognóstico assim como EEG inicial anormal correlacionar-se com prognóstico rescrvado, não houve diferença estatisticamente significativa entre os grupos aplicando-se o teste de Fischer.

\section{COMENTÁRIOS}

O EEG muitas vezes é o único meio de avaliar função cerebral em RN com patologias graves ou sob cfeito de medicaçōes, tendo-se tornado um método útil na avaliação destes bebês. $O$ seu valor na caracterização de patologias neurológicas neonatais e no prognóstico destes RN tem motivado o desenvolvimento de estudos envolvendo vários pesquisadores ${ }^{1.4-6.8-12}$.

Aso et col. ${ }^{1}$ revisando 180 traçados de $47 \mathrm{RN}$ que evoluíram para óbito e foram submetidos a necrópsia, observaram que o grau de anormalidade da atividade de base tem boa correlação com a severidade da lesão. Quanto mais alterado o EEG mais extensa e intensa foi a alteração morfológica encontrada.

Lombroso", correlacionando em exames seriados "maturidade" do EEG com IG e IC, observou que imaturidade transitória geralmente associa-se a bom prognóstico e imaturidade pcrsistente a prognóstico mais reservado.

Monod et col. ${ }^{6}$ estudando 691 EEGs de 270 RN com IG entre 36-38 semanas, observaram que EEGs normais obtidos na primeira semana de vida correlacionaram-se com bom prognóstico. Os achados como isoeletricidade, atividade paroxística, ausência de labilidade e organização espacial correlacionaram-se com mau prognóstico. Concluíram que as alterações eletrencefalograficas ajudam a predizer sequelas graves mas não auxiliam quando as sequelas são moderadas ou mínimas e sugerem que quanto mais precoce a realização do EEG melhor o seu valor prognóstico. 
Tharp et col. ". revisando $13 \mathrm{RN}$ prematuros com IG inferior a 36 semanas que foram submetidos a EEG seriados, observaram que EEGs seriados normais correlacionaram-se com bom prognóstico. Os achados como isocletricidade, atividade paroxística, ondas agudas positivas rolândicas, crise eletroclínica, assimetria de voltagem superior a $50 \%$, excesso de assincronia inter-hemisférica c ritmo lento com padrões inadequados para IC correlacionaram-se com prognóstico reservado.

Tharp et col. ${ }^{12}$ estudaram 62 RN prematuros com EEG seriado e seguimento de 2-3 anos. Sequelas neurológicas foram evidenciadas em todos os RN com alterações grosseiras do EEG (isoeletricidade, surto-supressão, intervalos intersurto prolongados e ondas agudas positivas rolândicas) e na maioria dos RN que apresentaram alteraçōes moderadas de forma persisitente (imaturidade, excessiva discontinuidade, excessiva assincronia inter-hemisférica, baixa voltagem ou focalidade). Observaram que EEGs persistentemente assimétricos estavam associados a patologias hemisféricas que determinavam hemi-sindromes. Concluíram que o EEG seriado no período neonatal ć melhor para predizer o prognóstico se comparado a neuroimagem e exame neurológico.

Nunes et col. ${ }^{8}$ revisando 56 RN com patologias neurológicas que foram submetidos a EEG/ PS e neuroimagem, observaram sensibilidade superior do EEG/PS e baixa especificidade quando relacionado a diagnóstico etiológico.

As ondas agudas positivas geralmente ocorrem relacionadas a patologias graves tais como leucomalácia e HIV², no nosso estudo quando presentes foram fortemente indicativas de mau prognóstico.

Considerando os estudos prévios que relacionam EEG, patologias neurológicas e prognóstico de $\mathrm{RN}$, nosso estudo apresentou seguimento médio de maior duração.

Nossos resultados concordam com os descritos na literatura quando comparamos alterações do EEG e prognóstico: isoeletricidade $\mathrm{e}$ atividade paroxística anormal (principalmente a presença de ondas agudas positivas) foram correlacionadas com seguimento anormal. Não foi possível relacionar com prognóstico as alterações como assimetria, imaturidade $\mathbf{e}$ baixa voltagem devido a perda de seguimento.

Relacionando patologia de base com EEG observamos que a imaturidade da atividade elétrica cerebral esteve presente como alteração mais frequente na maioria das patologias evidenciadas neste estudo (astixia, HIV. desnutriçăo intra-utero). Em estudo anterior ${ }^{\text {'b }} \mathrm{em}$ que foi avaliada uma população mista de RN prematuros e a termo com asfixia perinatal, já havíamos evidenciado esta associaçāo.

Não obtivemos diferença estatísticamente significativa entre os grupos quando comparamos de forma geral EEG normal ou altcrado com seguimento normal ou alterado. Este resultado provavelmente está relacionado ao fato de termos considerado neste estudo somentc o EEG inicial. A avaliaçāo EEG/PS seriada correlaciona-se melhor com prognóstico ${ }^{9,11,12}$.

\section{CONCLUSŌES}

Nossos resultados sugerem que:

1. As alteraçōes eletrencefalográficas do tipo isoelctricidade, atividade paroxística anormal (ondas agudás positivas) quando registraḍas em EEG de RN prematuros correlacionam-se com prognóstico reservado.

2. A alteração eletrencefalográfica mais freqüentemente encontrada em RN prematuros patológicos é a "imaturidade" da atividade elétrica cerebral.

3. O EEG/PS tem valor como coadjuvante na avaliação clínica e no prognóstico de RN prematuros com patologias neurológicas no período neonatal. 


\section{REFERÊNCIAS}

1. Aso K, Scher MS, Barmada MA. Neonatal electroencephalography and neuropathology. J Clin Neurophysiol 1989, 6:103-123.

2. Da Costa JC. O sono em recém-nascidos: aspectos polissonográficos. In: Reimão $R$ (ed) Sono: aspectos atuais. Rio de Janeiro: Atheneu, p 133-161.

3. Frankenburg WK, Dods JB. The Denver developmental screening test. J Pediatr 1967:181-191.

4. Lombroso CT. Neonatal EEG polygraphy in normal and abnormal newborns. In Niedermeyer, E, Lopes da Silva F (eds) Electroencephalography. Ed 3. Baltimore: Urban \& Schwarzenberg, 1994, p 803-875.

5. Lombroso CT. Neonatal polygraphy in full-term and premature infants: a review of normal and abnormal findings. J Clin Neurophysiol 1985, 2:105-155.

6. Monod N, Pajot N, Guidasci S. The neonatal EEG: statistical studies and prognostic value in full-term and pre-term babies. Electroenceph Clin Neurophysiol 1972, 32:529-544.

7. Nunes ML. Avaliaçāo da evolução da atividade elétrica cerebral em recém-nascidos através da polissonografia. Tese de Doutorado, (Universidade Estadual de Campinas), Campinas 1994.

8. Nunes ML, Faraco BM, Nora DB, Lopes DK, Brunstein M, Sperb J. Neuroradiology or neurophysiology: which method is the better approach to the diagnosis of neurologic diseases in the neonate? Pediatr Neurol 1992, 8:385.

9. Nunes ML, Da Costa JC, Godinho CL, Rodrigues MPR. Prognóstico de recém-nascidos com convulsões: aspectos clínicos e eletrencefalográficos. JLBE 1994, 7:27-30.

10. Silva EA. Nunes, ML, Da Costa, JC. Correlation of EEG and polysomnographic features with follow up in asphixiated newborns. Electroenceph Clin Neurophysiol 1993, 2:(Suppl).

11. Tharp BR, Cukicr F, Monod N. The prognostic value of the electroencephalogram in premature infants. Electroenceph Clin Neurophysiol 1981, 51:219-236.

12. Tharp BR, Scher MS, Clancy RR. Serial EEGs in normal and abnormal infants with birth weights less than 1200 grams: a prospective study with long-term follow-up. Neuropediatrics 1989, 20:64-72. 